\title{
Resistance to Ciprofloxacin in urinary infections in patients hospitalized in the medicinainterna service
}

\begin{abstract}
Introduction: Urinary tract infections (UTI) are one of the most frequent infectious processes worldwide. The estimated incidence of UTI in our country is currently unknown. The treatment will depend on the age, the sex, the isolated germ, among others. Fluoroquinolones have been considered as the ideal treatment in this infection. The most empirically used is ciprofloxacin; however, its resistance makes a marked decrease in the effectiveness of the treatment with this agent.
\end{abstract}

Objective: To describe the microbial resistance to ciprofloxacin in UTI as first-line treatment.

Methods: Descriptive, cross-sectional and retrospective study, through the review of clinical records of 217 patients, who had the diagnosis of UTI, with reported cultures, during the period January 2013 to December 2017.

Results: 82 patients were included $(37.76 \%)$. The main germs that reported resistance were Escherichia coli (54.23\%), Pseudomonas aeruginosa $(11.86 \%)$. The most frequent comorbidity was Diabetes Mellitus. And the bacterial resistance reported in this study is $79.66 \%$

Discussion: Resistance to ciprofloxacin constitutes a serious public health problem due to its frequent use. E coli were the bacterium with the greatest resistance to this antibiotic, superior to the reviewed studies. The proportion of resistance exceeds that reported in other works.

Conclusion: Resistance to ciprofloxacin in urinary tract infection caused by common uropathogens is increasing. The use of this antibiotic agent as empirical therapy for UTI should be reconsidered and efforts should be made to limit its use to clinical conditions that warrant it.
Volume 7 Issue 2 - 2019

\author{
Erika Arismendi,'2 Ray Molina,' Michelle \\ Aldama,' Magaly Barazarte, ${ }^{2}$ Paulibeth \\ Alvarado,' José Rivas' \\ 'School of Medicine, Universidad Nacional Experimental \\ Francisco de Miranda, Venezuela \\ ${ }^{2}$ Internist, deputy to the Internal Medicine Service, University \\ Hospital “Dr. Pedro Emilio Carrillo”, Venezuela
}

\section{Correspondence: Ray Molina, Medical Student, School of Medicine, Universidad Nacional Experimental Francisco de Miranda, Coro, Venezuela, Tel +584I68782744,}

Email raydavidm25@gmail.com

Received: March 05, 2019 | Published: April 24, 2019

Keywords: Ciprofloxacin, urinary tract infection, microbial resistance

\section{Introduction}

Urinary tract infections (UTI) are considered as one of the most frequent infectious processes worldwide, representing a high prevalence in the field of public health, due to the statistical increase of healthy people and people with comorbidity who suffer from it, especially due to potential complications such as kidney injury, urinary sepsis, microbial resistance, among others, which directly affect the healthy lifestyle of patients, producing a high cost and impact on health services. The estimated incidence of UTI in our country is currently unknown. However, it is believed that this phenomenon is repeated in other developing countries. ${ }^{1}$ UTIs represent more than 100,000 hospitalizations per year in countries such as the United States, most of them due to complicated UTIs. Likewise, they represent approximately $40 \%$ of all nosocomial infections, which in most cases, are associated with placement of bladder catheters. ${ }^{1,2}$

Clinically, UTI can go from uncomplicated to complicated. Treatment will depend on age, sex, isolated germ, comorbidities and the location of the infection in the urinary tract. ${ }^{3}$ By For a long time, fluoroquinolones have been considered as the antibiotics most frequently prescribed in nosocomial UTIs and acquired in the community. Being one of the first groups of antibiotics indicated empirically, its microbial resistance represents

serious clinical, epidemiological and public health complications. ${ }^{4}$ Many publications, from different nations, have shown positive evidence that bacterial resistance to antibiotics is related to several factors such as self-medication or erroneous prescription in non-merit antibiotic clinical situations. ${ }^{5,6}$

The fluoroquinolone most empirically used in ITU has always been Ciprofloxacin, due to the benefits it has in its pharmacodynamics, which make it more preferred in its use in uncomplicated and complicated infections; It also has a good spectrum for gram-negative bacteria. However, the increase of microbial resistance on the part of the uropathogens makes a marked decrease in the effectiveness of the Ciprofloxacin treatments, to this is added the increase in the prevalence of pathogenic bacteria producing extended-spectrum betalactamases (ESBL). What supposes a greater resistance to antibiotics. ${ }^{7}$ Therefore, it is important to describe the microbial resistance to Ciprofloxacin as a first-line treatment in urinary tract infections, as well as to identify the factors related to this resistance, in order to establish bases to improve the selection and prescription of an appropriate antibiotic in patients with urinary tract infection.

\section{Material and methods}

Descriptive, cross-sectional and retrospective study, through the review of clinical records of 217 patients, as a universe, which had the diagnosis of Urinary Tract Infection, with reported cultures. The study was carried out in the Internal Medicine Service of the University Hospital "Dr. Pedro Emilio Carrillo "de Valera, Trujillo, Venezuela, together with the file and record of medical records attached to the hospital. 
Inclusion criteria: Patients hospitalized in the Internal Medicine Service with a diagnosis of urinary tract infection during the period January 2013 to December 2017. Urine cultures that report antibiogram with resistance and sensitivity.

Exclusion criteria: Previous hospitalization in another medical service. No report of urine cultures with antibiogram.

A systematic review of the clinical histories was carried out together with the urine cultures, to the patients who met the inclusion criteria in order to collect the data. The majority of the clinical records (urocultures) reviewed correspond to samples taken by the patients themselves and processed in private clinical laboratories through tests of bacterial sensitivity through the E-test method. The information is presented using descriptive statistics, describing the frequency of ciprofloxacin resistance report in urinary infection of the aforementioned hospitalized patients.

\section{Results}

Of all the data reviewed, with 217 selected patients, 82 (37.76\%) were included who fulfilled the study criteria, all of different ages, of both sexes, hospitalized whose distribution is described in Table 1; of these, $59(71.95 \%)$ had positive urine cultures with antibiogram reporting sensitivity and resistance to antibiotics, especially Ciprofloxacin, and 23 (28.04\%), did not report bacterial growth.

Table I Age of patients who underwent cultures

\begin{tabular}{llll}
\hline Total of Urocultivos & Average age & Median & Media \\
\hline$n=82$ & 49 & 52 & 59 \\
\hline
\end{tabular}

Of the analyzed urine cultures $100 \%$ were monomicrobial. The main Germs, in order of frequency, that reported resistance to Ciprofloxacin were Escherichia coli in 32 episodes (54.23\%), Pseudomonas aeruginosa 7 (11.86\%), Proteus vulgaris 3 (5.08\%), Enterobacter spp $2(3.38 \%)$. The rest of the isolated microorganisms are described in Table 2. The incidence of ESBL producing Uropathogenic strains was $31.25 \%$ of the total cultivated $E$. coli strains and $28.57 \%$ of $P$. aeruginosa.

Table 2 Frequency of uropathogens reported

\begin{tabular}{lll}
\hline Germ & Frequency & Percentage \\
\hline E Coli & 32 & 54,23 \\
Candida spp & 9 & 14,25 \\
Pseudomona aeruginosa & 7 & 11,86 \\
Proteus vulgaris & 3 & 5,08 \\
Enterobacter spp & 2 & 3,38 \\
Proteus spp & 2 & 3,38 \\
Staphylococcus aureus & $\mathrm{I}$ & 1,69 \\
Staphylococcus saprophyticus & $\mathrm{I}$ & 1,69 \\
\hline
\end{tabular}

*Candida spp does not include the sample of the first three pathogenic microorganisms because it belongs to the Fungi kingdom, in such a way that it does not comply with the objective of this research work.

Table 3 shows the comorbidities associated with the patients studied; the three main ones were: Diabetes Mellitus in 35 patients (42.68\%), renal lithiasis 21 (25.66) and infection by retrovirus $3(3.65 \%)$. It was found that about $15 \%$ of the patients studied underwent bladder catheterization with a frequency of $P$. aeruginosa and Staphylococcus saprophyticus in the cases reported.
The resistance to Ciprofloxacin reported in the antibiograms of the isolated germs is $79.66 \%$ in 47 cases, as shown in Table 4 .

Table 3 Associated comorbidities

\begin{tabular}{lll}
\hline Total patients & Comorbidities & Percentage \\
\hline $\mathrm{n}=82$ & & \\
35 & Diabetes mellitus & 44,44 \\
21 & Nefrolitiasis & $42,5 \mathrm{I}$ \\
3 & Retrovirus infection & 2,89 \\
3 & Epilepsia & 2,89 \\
2 & Bronchial asthma & $2,4 \mathrm{I}$ \\
2 & ITU previa & $2,4 \mathrm{I}$ \\
I & Raquimedular trauma & $\mathrm{I}, 44$ \\
\hline
\end{tabular}

Table 4 Resistance to Ciprofloxacin

\begin{tabular}{ll}
\hline Resistance to Ciprofloxacin & Percentage \\
\hline Resistance & $47(79,66 \%)$ \\
Sensitivity & $2(3,38 \%)$ \\
Not reported & $10(16,94 \%)$ \\
Total & $59(99,98)$ \\
\hline
\end{tabular}

\section{Discussion}

Previous research studies reported a microbial resistance to Ciprofloxacin, with an average of $35 \%$. However, the present investigation shows a considerable increase in resistance, by common uropathogens, with a higher incidence in gram-negative enterobacteria; therefore, it becomes evident that as the intrinsic mechanisms of the bacteria advance to generate resistance to the quinolones, they will soon cease to be a valid treatment option.

A diversity of isolated germs was evidenced, being Escherichia coli the bacterial agent with greater isolation in $54.23 \%$, followed by Pseudomonas aeruginosa with 7 and Proteus vulgaris with 3 cases, in addition it was observed that in all cases they were monomicrobial infections. In the same way, the incidence of ESBL-producing strains was reported from $E$. coli isolates and of $P$. aeruginosa, in such a way that it differs from the results of the study published by Sanjida ${ }^{8}$ where the isolated microbiology with more frequency were Escherichia coli (63.93\%) followed by Klebsiella pneumoniae (17.09\%), and where reported ESBL strains.

When evaluating the population studied, it was obtained that 35 of the patients presented Diabetes Mellitus as the main comorbidity, followed by nephrolithiasis with 21 documented cases. Therefore, it is analyzed and compared that diabetes mellitus remains the main comorbidity associated with the appearance of urinary tract infection, demonstrating the congruence in the report of Alberto, ${ }^{9}$ where it is stated that diabetes mellitus is the first, although not the only risk factor for the acquisition of a UTI at the intra and extrahospitalary level. The resistance to ciprofloxacin in the total of antibiograms for the isolated germs, has a greater report on the part of the gramnegative bacteria to said antibiotic, above the $79 \%$, being high in comparison with the data obtained in $\mathrm{Jae}^{7}$ work, which showed levels of resistance to Ciprofloxacin of $15.2 \%$. As could be analyzed in this study, resistance to this antibiotic could be due to its massive use in clinical practice, its broad spectrum of action and pharmacokinetic 
properties; in contrast, a plausible explanation for the lower resistance found in other groups of antimicrobial agents such as amikacin or nitrofurantoin, could be the fact of cost and low adherence that limits its use in our environment, manifesting in the observed results.

\section{Conclusion}

The increasing increase in the resistance of this antibiotic, the high costs in the pharmacological treatments, are only 2 relevant aspects that justify the study of the unprecedented increase of antimicrobials that are not very effective due to the microbial resistance in Venezuela and in the region. It is important to teach microbial resistance to professionals in the Health Sciences Area, both at undergraduate and postgraduate levels, in order to promote the accurate diagnosis of common infections such as UTI, preparing and encouraging the correct indication of antibiotic treatment. Resistance to ciprofloxacin in urinary tract infection caused by common uropathogens is increasing. The use of this antibiotic agent as empirical therapy for UTI should be reconsidered and efforts should be made to limit its use to clinical conditions that warrant it. More research is needed to describe the resistance to ciprofloxacin. Therefore, it is recommended to continue with the present research work, as a line and basis for future broad and inclusive studies, with the aim of extending and updating the accurate and scientific knowledge about urinary infections and the production of resistance to the different antimicrobial agents, as well as take them to a greater number of health centers.

\section{Acknowledgments}

None.

\section{Conflicts of interest}

Authors declare that there is no conflict of interest.

\section{References}

1. Borregales L, Giordano F, Contreras L. Definitions of Urinary Infections. Caracas: SVU; 2011.

2. Pickard R, Bartoletti R, Bjerklund-Johansen TE, et al. EAU Guidelines on Urological Infections. European Association of Urology; 2016. 16 p.

3. Gupta K, Hooton TM, Naber KG, et al. International Clinical practice guidelines for the treatment of acute uncomplicated cystitis and pyelonephritis in women. Clinical Infectious Diseases. 2011;52(5):e103e120.

4. Smith D, Tanagho E. Urología General de Smith. 10th ed. San Francisco: MARBAN; 2015.

5. Gallardo Luna MG, Magraña Aquino M, Andrade Rodríguez HJ, et al. Antimicrobial resistance amoing them employed in urinary tract infections in primary care patients from a familiar medicina unit from the IMSS. Enfermedades Infecciosas y Microbiologia. 2008;28(1):13-18.

6. Fariña N, Sanabria R. Actividad invitro de fluoroquinolonas en Bacilos Gramnegativos aislados de urocultivos de pacientes ambiulatorios. Mem Inst Investig Cienc Salud. 2007;5(1):15-18.

7. Jeon JH, Kim K, Han WD, et al. Empirical use of Ciprofloxacin for acute uncomplicated pyelonephritis caused by Escherichia coli in communities where the prevalence of fluoroquinolone resistence is high. Antimicrob Agents Chemother. 2012;56(6):3043-3046.

8. Khondakar S, Ibne AN, Saleh A, et al. Study of Bacterial pathogens in Urinary Tract Infection and their antibiotic resistance profile in a tertiary care hospital of Bangladesh. Bangladesh J Med Microbiol. 2016;10(1):22-26.

9. Pedraza A, Dávila R, Acevedo O, et al. Urinary tract infection: prevalence, antimicrobial resistence and associated ricks factors in type 2 diabetes mellitus patients. Rev Cubana Endocrinol. 2014;25(2):57-65. 